\title{
Convergence of a regularization algorithm for nonexpansive and monotone operators in Hilbert spaces
}

Qing Yuan ${ }^{1}$ and Yunpeng Zhang ${ }^{2^{*}}$

\section{"Correspondence:}

zhangypliyl@yeah.net

${ }^{2}$ Department of Mathematics,

Jinming Institute of Education,

Jinming, China

Full list of author information is

available at the end of the article

\begin{abstract}
Variational inequality, fixed point and generalized equilibrium problems are investigated via a regularization algorithm. It is proved that the sequence generated in the regularization algorithm converges strongly to a common solution of the three problems in the framework of Hilbert spaces. The results presented in this paper improve and extend the corresponding ones announced by many authors.
\end{abstract}

Keywords: equilibrium problem; fixed point; variational inequality; zero point

\section{Introduction and preliminaries}

In this paper, we always assume that $H$ is a real Hilbert space with inner product $\langle x, y\rangle$ and induced norm $\|x\|=\sqrt{\langle x, x\rangle}$ for $x, y \in H$. Let $C$ be a nonempty, closed, and convex subset of $H$.

Let $A: C \rightarrow H$ be a mapping. Recall that $A$ is said to be monotone iff

$$
\langle A x-A y, x-y\rangle \geq 0, \quad \forall x, y \in C .
$$

Recall that $A$ is said to be strongly monotone iff there exists a constant $\alpha>0$ such that

$$
\langle A x-A y, x-y\rangle \geq \alpha\|x-y\|^{2}, \quad \forall x, y \in C .
$$

For such a case, $A$ is also said to be $\alpha$-strongly monotone. Recall that $A$ is said to be inversestrongly monotone iff there exists a constant $\alpha>0$ such that

$$
\langle A x-A y, x-y\rangle \geq \alpha\|A x-A y\|^{2}, \quad \forall x, y \in C .
$$

For such a case, $A$ is also said to be $\alpha$-inverse-strongly monotone.

Recall that the classical variational inequality is to find an $x \in C$ such that

$$
\langle A x, y-x\rangle \geq 0, \quad \forall y \in C
$$

In this paper, we always use $V I(C, A)$ to denote the solution set of $(1.1)$ and use $P_{C}$ denote the metric projection from $H$ onto $C$. It is well known that $x \in C$ is a solution of (1.1)

\section{Springer}

(c) 2014 Yuan and Zhang; licensee Springer. This is an Open Access article distributed under the terms of the Creative Commons Attribution License (http://creativecommons.org/licenses/by/2.0), which permits unrestricted use, distribution, and reproduction in any medium, provided the original work is properly cited. 
iff $x$ is a fixed point of the mapping $P_{C}(I-r A)$, where $r>0$ is a constant, $I$ stands for the identity mapping. If $A$ is strongly monotone and Lipschitz continuous, the existence and uniqueness of solutions of equilibrium (1.1) is guaranteed by the Banach contraction principle.

Recall that a set-valued mapping $M: H \rightrightarrows H$ is said to be monotone iff, for all $x, y \in H$, $f \in M x$, and $g \in M y$ imply $\langle x-y, f-g\rangle>0 . M$ is maximal iff the graph $\operatorname{Graph}(M)$ of $R$ is not properly contained in the graph of any other monotone mapping. It is well known that a monotone mapping $M$ is maximal if and only if, for any $(x, f) \in H \times H,\langle x-y, f-g\rangle \geq 0$, for all $(y, g) \in \operatorname{Graph}(M)$ implies $f \in R x$.

Let $S: C \rightarrow C$ be a mapping. $F(S)$ stands for the fixed point set of $S$; that is, $F(S):=\{x \in$ $C: x=S x\}$.

Recall that $S$ is said to be contractive iff there exists a constant $\alpha \in(0,1)$ such that

$$
\|S x-S y\| \leq \alpha\|x-y\|, \quad \forall x, y \in C .
$$

For such a case, $S$ is also said to be $\alpha$-contractive. We know that the mapping enjoys a unique fixed point and Picard's algorithm can be employed to approximate its unique fixed point.

Recall that $S$ is said to be nonexpansive iff

$$
\|S x-S y\| \leq\|x-y\|, \quad \forall x, y \in C .
$$

If $C$ is a closed, bounded and convex subset of $H$, then $F(S)$ is not empty; see [1].

Let $\left\{S_{i}: C \rightarrow C\right\}$ be a family of infinitely nonexpansive mappings and $\left\{\gamma_{i}\right\}$ be a nonnegative real sequence with $0 \leq \gamma_{i}<1, \forall i \geq 1$. For $n \geq 1$, define a mapping $W_{n}: C \rightarrow C$ as follows:

$$
\begin{aligned}
& U_{n, n+1}=I, \\
& U_{n, n}=\gamma_{n} S_{n} U_{n, n+1}+\left(1-\gamma_{n}\right) I, \\
& U_{n, n-1}=\gamma_{n-1} S_{n-1} U_{n, n}+\left(1-\gamma_{n-1}\right) I, \\
& \vdots \\
& U_{n, k}=\gamma_{k} S_{k} U_{n, k+1}+\left(1-\gamma_{k}\right) I, \\
& U_{n, k-1}=\gamma_{k-1} S_{k-1} U_{n, k}+\left(1-\gamma_{k-1}\right) I, \\
& \vdots \\
& U_{n, 2}=\gamma_{2} S_{2} U_{n, 3}+\left(1-\gamma_{2}\right) I, \\
& W_{n}=U_{n, 1}=\gamma_{1} S_{1} U_{n, 2}+\left(1-\gamma_{1}\right) I .
\end{aligned}
$$

Such a mapping $W_{n}$ is nonexpansive from $C$ to $C$ and it is called a $W$-mapping generated by $S_{n}, S_{n-1}, \ldots, S_{1}$ and $\gamma_{n}, \gamma_{n-1}, \ldots, \gamma_{1}$; see [2] and the references therein.

Let $T: C \rightarrow H$ be a monotone mapping and let $F$ be a bifunction of $C \times C$ into $\mathbb{R}$, where $\mathbb{R}$ denotes the set of real numbers. We consider the following generalized equilibrium 
problem:

$$
\text { Find } x \in C \text { such that } F(x, y)+\langle T x, y-x\rangle \geq 0, \quad \forall y \in C \text {. }
$$

In this paper, the set of such $x \in C$ is denoted by $E P(F, T)$, i.e.,

$$
\operatorname{GEP}(F, A)=\{x \in C: F(x, y)+\langle T x, y-x\rangle \geq 0, \forall y \in C\} .
$$

If $T \equiv 0$, the zero mapping, then the problem (1.3) is reduced to the following equilibrium problem [3]:

$$
\text { Find } x \in C \text { such that } F(x, y) \geq 0, \quad \forall y \in C \text {. }
$$

In this paper, the set of such an $x \in C$ is denoted by $E P(F)$.

If $F \equiv 0$, then the problem (1.3) is reduced to the classical variational inequality (1.1).

To study equilibrium problems (1.3) and (1.4), we may assume that $F$ satisfies the following conditions:

(A1) $F(x, x)=0$ for all $x \in C$;

(A2) $F$ is monotone, i.e., $F(x, y)+F(y, x) \leq 0$ for all $x, y \in C$;

(A3) for each $x, y, z \in C$,

$$
\limsup _{t \downarrow 0} F(t z+(1-t) x, y) \leq F(x, y) ;
$$

(A4) for each $x \in C, y \mapsto F(x, y)$ is convex and weakly lower semi-continuous.

Many important problems have reformulations which require finding solutions of equilibriums (1.3) and (1.4), for instance, image recovery, inverse problems, network allocation, transportation problems and optimization problems; see [3-11] and the references therein. For solving solutions of equilibriums (1.3) and (1.4), regularization methods recently have been extensively studied; see [11-28] and the references therein.

In this paper, motivated and inspired by the research going on in this direction, we study the variational inequality (1.1), and the fixed point and equilibrium problem (1.3) based on a regularization algorithm. It is proved that the sequence generated in the regularization algorithm converges strongly to a common solutions of the three problems in the framework of Hilbert spaces. The results presented in this paper improve and extend the corresponding results in Chang et al. [11], Takahashi and Takahashi [13] and Hao [29].

The following lemmas play an important role in our paper.

Lemma 1.1 [3] Let $F: C \times C \rightarrow \mathbb{R}$ be a bifunction satisfying (A1)-(A4). Then, for any $r>0$ and $x \in H$, there exists $z \in C$ such that

$$
F(z, y)+\frac{1}{r}\langle y-z, z-x\rangle \geq 0, \quad \forall y \in C .
$$

Define a mapping $T_{r}: H \rightarrow C$ as follows:

$$
T_{r} x=\left\{z \in C: F(z, y)+\frac{1}{r}\langle y-z, z-x\rangle \geq 0, \forall y \in C\right\}, \quad x \in H,
$$

then the following conclusions hold: 
(1) $T_{r}$ is single-valued;

(2) $T_{r}$ is firmly nonexpansive, i.e., for any $x, y \in H$,

$$
\left\|T_{r} x-T_{r} y\right\|^{2} \leq\left\langle T_{r} x-T_{r} y, x-y\right\rangle
$$

(3) $F\left(T_{r}\right)=E P(F)$;

(4) $E P(F)$ is closed and convex.

Lemma 1.2 [30] Assume that $\left\{\alpha_{n}\right\}$ is a sequence of nonnegative real numbers such that

$$
\alpha_{n+1} \leq\left(1-\gamma_{n}\right) \alpha_{n}+\delta_{n}
$$

where $\left\{\gamma_{n}\right\}$ is a sequence in $(0,1)$ and $\left\{\delta_{n}\right\}$ is a sequence such that

(1) $\sum_{n=1}^{\infty} \gamma_{n}=\infty$;

(2) $\lim \sup _{n \rightarrow \infty} \delta_{n} / \gamma_{n} \leq 0$ or $\sum_{n=1}^{\infty}\left|\delta_{n}\right|<\infty$.

Then $\lim _{n \rightarrow \infty} \alpha_{n}=0$.

Lemma 1.3 [2] Let $\left\{S_{i}: C \rightarrow C\right\}$ be a family of infinitely nonexpansive mappings with a nonempty common fixed point set and let $\left\{\gamma_{i}\right\}$ be a real sequence such that $0<\gamma_{i} \leq l<1$, where $l$ is some real number, $\forall i \geq 1$. Then

(1) $W_{n}$ is nonexpansive and $F\left(W_{n}\right)=\bigcap_{i=1}^{\infty} F\left(S_{i}\right)$, for each $n \geq 1$;

(2) for each $x \in C$ and for each positive integer $k$, the limit $\lim _{n \rightarrow \infty} U_{n, k}$ exists.

(3) the mapping $W: C \rightarrow C$ defined by

$$
W x:=\lim _{n \rightarrow \infty} W_{n} x=\lim _{n \rightarrow \infty} U_{n, 1} x, \quad x \in C,
$$

is a nonexpansive mapping satisfying $F(W)=\bigcap_{i=1}^{\infty} F\left(S_{i}\right)$ and it is called the $W$-mapping generated by $S_{1}, S_{2}, \ldots$ and $\gamma_{1}, \gamma_{2}, \ldots$

Lemma 1.4 [31] Let $\left\{x_{n}\right\}$ and $\left\{y_{n}\right\}$ be bounded sequences in $H$ and let $\left\{\beta_{n}\right\}$ be a sequence in $(0,1)$ with $0<\liminf _{n \rightarrow \infty} \beta_{n} \leq \limsup _{n \rightarrow \infty} \beta_{n}<1$. Suppose that $x_{n+1}=\left(1-\beta_{n}\right) y_{n}+\beta_{n} x_{n}$ for all $n \geq 0$ and

$$
\limsup _{n \rightarrow \infty}\left(\left\|y_{n+1}-y_{n}\right\|-\left\|x_{n+1}-x_{n}\right\|\right) \leq 0 \text {. }
$$

Then $\lim _{n \rightarrow \infty}\left\|y_{n}-x_{n}\right\|=0$.

Lemma 1.5 [11] Let $\left\{S_{i}: C \rightarrow C\right\}$ be a family of infinitely nonexpansive mappings with a nonempty common fixed point set and let $\left\{\gamma_{i}\right\}$ be a real sequence such that $0<\gamma_{i} \leq l<1$, $\forall i \geq 1$. If $K$ is any bounded subset of $C$, then

$$
\lim _{n \rightarrow \infty} \sup _{x \in K}\left\|W x-W_{n} x\right\|=0 .
$$

Throughout this paper, we always assume that $0<\gamma_{i} \leq l<1, \forall i \geq 1$. 
Lemma 1.6 [10] Let $A: C \rightarrow H$ a Lipschitz monotone mapping and let $N_{C} x$ be the normal cone to $C$ at $x \in C$; that is, $N_{C} x=\{y \in H:\langle x-u, y\rangle, \forall u \in C\}$. Define

$$
D x= \begin{cases}A x+N_{C} x, & x \in C, \\ \varnothing & x \notin C .\end{cases}
$$

Then $D$ is maximal monotone and $0 \in D x$ if and only if $x \in V I(C, A)$.

\section{Main results}

Theorem 2.1 Let $C$ be a nonempty closed convex subset of $H$. Let $F$ be a bifunction from $C \times C$ to $\mathbb{R}$ which satisfies (A1)-(A4) and let $f: C \rightarrow C$ be a $\kappa$-contraction. Let $A: C \rightarrow H$ be an $\alpha$-inverse-strongly monotone mapping and let $B: C \rightarrow H$ be a $\beta$-inverse-strongly monotone mapping. Let $T: C \rightarrow H$ be a $\tau$-inverse-strongly monotone mapping. Let $\left\{S_{i}\right.$ : $C \rightarrow C$ \} be a family of infinitely nonexpansive mappings. Assume that $\Sigma=\bigcap_{i=1}^{\infty} F\left(S_{i}\right) \cap$ $\operatorname{GEP}(F, T) \cap V I(C, A) \cap V I(C, B)$ is not empty. Let $\left\{\alpha_{n}\right\},\left\{\beta_{n}\right\}$, and $\left\{\gamma_{n}\right\}$ be sequences in $(0,1)$ such that $\alpha_{n}+\beta_{n}+\gamma_{n}=1$. Let $\left\{r_{n}\right\},\left\{s_{n}\right\}$, and $\left\{\lambda_{n}\right\}$ be positive number sequences. Let $x_{1} \in C$ and let $\left\{x_{n}\right\}$ be a sequence generated by

$$
\left\{\begin{array}{l}
y_{n}=P_{C}\left(u_{n}-s_{n} B u_{n}\right), \\
x_{n+1}=\alpha_{n} f\left(y_{n}\right)+\beta_{n} W_{n} P_{C}\left(y_{n}-r_{n} A y_{n}\right)+\gamma_{n} x_{n}, \quad \forall n \geq 1,
\end{array}\right.
$$

where $\left\{u_{n}\right\}$ is such that $F\left(u_{n}, y\right)+\left\langle T x_{n}, y-u_{n}\right\rangle+\frac{1}{\lambda_{n}}\left\langle y-u_{n}, u_{n}-x_{n}\right\rangle \geq 0, \forall y \in C$, and $\left\{W_{n}\right\}$ is the sequence generated in (1.5). Assume that the following restrictions hold:

(a) $0<a \leq \lambda_{n} \leq b<2 \tau$ and $\lim _{n \rightarrow \infty}\left|\lambda_{n+1}-\lambda_{n}\right|=0$,

(b) $0<a^{\prime} \leq r_{n} \leq b^{\prime}<2 \alpha$ and $\lim _{n \rightarrow \infty}\left|r_{n+1}-r_{n}\right|=0$,

(c) $0<a^{\prime \prime} \leq s_{n} \leq b^{\prime \prime}<2 \beta$ and $\lim _{n \rightarrow \infty}\left|s_{n+1}-s_{n}\right|=0$,

(d) $\lim _{n \rightarrow \infty} \alpha_{n}=0, \sum_{n=1}^{\infty} \alpha_{n}=\infty$,

(e) $0<\liminf _{n \rightarrow \infty} \gamma_{n} \leq \limsup _{n \rightarrow \infty} \gamma_{n}<1$,

where $a, a^{\prime}, a^{\prime \prime}, b, b^{\prime}$, and $b^{\prime \prime}$ are real constants. Then $\left\{x_{n}\right\}$ converges strongly to $\bar{x} \in \Sigma$, which solves uniquely the following variational inequality:

$$
\langle\bar{x}-f(\bar{x}), \bar{x}-x\rangle \leq 0, \quad \forall x \in \Sigma .
$$

Proof Since $A$ is inverse-strongly monotone, we see from restriction (b) that

$$
\begin{aligned}
\|( & \left.-r_{n} A\right) x-\left(I-r_{n} A\right) y \|^{2} \\
& =\|x-y\|^{2}-2 r_{n}\langle x-y, A x-A y\rangle+r_{n}^{2}\|A x-A y\|^{2} \\
& \leq\|x-y\|^{2}-2 r_{n} \alpha\|A x-A y\|^{2}+r_{n}^{2}\|A x-A y\|^{2} \\
& =\|x-y\|^{2}+r_{n}\left(r_{n}-2 \alpha\right)\|A x-A y\|^{2} \\
& \leq\|x-y\|^{2}, \quad \forall x, y \in C .
\end{aligned}
$$

This shows that $I-r_{n} A$ is nonexpansive. In the same way, we find that $I-s_{n} B$ and $I-\lambda_{n} T$ are nonexpansive. Note that $u_{n}$ can be re-written as $u_{n}=T_{\lambda_{n}}\left(I-\lambda_{n} T\right) x_{n}$. Let $x^{*} \in \Sigma$. It 
follows that

$$
\left\|u_{n}-x^{*}\right\| \leq\left\|\left(I-\lambda_{n} T\right) x_{n}-\left(I-\lambda_{n} T\right) x^{*}\right\| \leq\left\|x_{n}-x^{*}\right\| .
$$

Putting $z_{n}=P_{C}\left(y_{n}-r_{n}\right) A y_{n}$, we see that $\left\|z_{n}-x^{*}\right\| \leq\left\|y_{n}-x^{*}\right\| \leq\left\|x_{n}-x^{*}\right\|$.

$$
\begin{aligned}
\left\|x_{n+1}-x^{*}\right\| & \leq \alpha_{n}\left\|f\left(y_{n}\right)-x^{*}\right\|+\beta_{n}\left\|W_{n} z_{n}-x^{*}\right\|+\gamma_{n}\left\|x_{n}-x^{*}\right\| \\
& \leq \alpha_{n} \kappa\left\|y_{n}-x^{*}\right\|+\alpha_{n}\left\|f\left(x^{*}\right)-x^{*}\right\|+\beta_{n}\left\|z_{n}-x^{*}\right\|+\gamma_{n}\left\|x_{n}-x^{*}\right\| \\
& \leq\left(1-\alpha_{n}(1-\kappa)\right)\left\|x_{n}-x^{*}\right\|+\alpha_{n}(1-\kappa) \frac{\left\|f\left(x^{*}\right)-x^{*}\right\|}{1-\kappa} .
\end{aligned}
$$

This implies that

$$
\left\|x_{n}-x^{*}\right\| \leq \max \left\{\left\|x_{1}-x^{*}\right\|, \frac{\left\|f\left(x^{*}\right)-x^{*}\right\|}{1-\kappa}\right\}<\infty .
$$

This yields the result that the sequence $\left\{x_{n}\right\}$ is bounded, and so are $\left\{y_{n}\right\},\left\{z_{n}\right\}$, and $\left\{u_{n}\right\}$. Without loss of generality, we can assume that there exists a bounded set $K \subset C$ such that $x_{n}, y_{n}, z_{n}, u_{n} \in K$. Since $u_{n}=T_{\lambda_{n}}\left(I-\lambda_{n}\right) x_{n}$, we find that

$$
F\left(u_{n+1}, y\right)+\frac{1}{\lambda_{n+1}}\left\langle y-u_{n+1}, u_{n+1}-\left(I-r_{n+1} T\right) x_{n+1}\right\rangle \geq 0, \quad \forall y \in C,
$$

and

$$
F\left(u_{n}, y\right)+\frac{1}{\lambda_{n}}\left\langle y-u_{n}, u_{n}-\left(I-\lambda_{n} T\right) x_{n}\right\rangle \geq 0, \quad \forall y \in C .
$$

Let $y=u_{n}$ in (2.2) and $y=u_{n+1}$ in (2.3). By adding up these two inequalities, we obtain

$$
\left\langle u_{n+1}-u_{n}, u_{n}-u_{n+1}+u_{n+1}-\left(I-\lambda_{n} A_{3}\right) x_{n}-\frac{\lambda_{n}}{\lambda_{n+1}}\left(u_{n+1}-\left(I-\lambda_{n+1} A_{3}\right) x_{n+1}\right)\right\rangle \geq 0 .
$$

This implies that

$$
\begin{aligned}
\left\|u_{n+1}-u_{n}\right\|^{2} \leq & \left\langle u_{n+1}-u_{n},\left(I-\lambda_{n+1} T\right) x_{n+1}-\left(I-\lambda_{n} T\right) x_{n}\right. \\
& \left.+\left(1-\frac{\lambda_{n}}{\lambda_{n+1}}\right)\left(u_{n+1}-\left(I-\lambda_{n+1} T\right) x_{n+1}\right)\right\rangle \\
\leq & \left\|u_{n+1}-u_{n}\right\|\left(\left\|\left(I-\lambda_{n+1} T\right) x_{n+1}-\left(I-\lambda_{n} T\right) x_{n}\right\|\right. \\
& \left.+\left|1-\frac{\lambda_{n}}{\lambda_{n+1}}\right|\left\|u_{n+1}-\left(I-\lambda_{n+1} T\right) x_{n+1}\right\|\right) .
\end{aligned}
$$

It follows that

$$
\begin{aligned}
\left\|u_{n+1}-u_{n}\right\| \leq & \left\|\left(I-\lambda_{n+1} T\right) x_{n+1}-\left(I-\lambda_{n} T\right) x_{n}\right\| \\
& +\frac{\left|\lambda_{n+1}-\lambda_{n}\right|}{\lambda_{n+1}}\left\|u_{n+1}-\left(I-\lambda_{n+1} T\right) x_{n+1}\right\| \\
\leq & \left\|x_{n+1}-x_{n}\right\|+\left|\lambda_{n+1}-\lambda_{n}\right| M_{1}
\end{aligned}
$$


where $M_{1}$ is an appropriate constant such that

$$
M_{1}=\sup _{n \geq 1}\left\{\left\|T x_{n}\right\|+\frac{\left\|u_{n+1}-\left(I-\lambda_{n+1} T\right) x_{n+1}\right\|}{a}\right\} .
$$

It follows from (2.4) that

$$
\begin{aligned}
\left\|y_{n+1}-y_{n}\right\| \leq & \left\|P_{C}\left(u_{n+1}-s_{n+1} B u_{n+1}\right)-P_{C}\left(u_{n}-s_{n+1} B u_{n}\right)\right\| \\
& +\left\|P_{C}\left(u_{n}-s_{n+1} B u_{n}\right)-P_{C}\left(u_{n}-s_{n} B u_{n}\right)\right\| \\
\leq & \left\|u_{n+1}-u_{n}\right\|+\left|s_{n+1}-s_{n}\right|\left\|B u_{n}\right\| \\
\leq & \left\|x_{n+1}-x_{n}\right\|+\left|\lambda_{n+1}-\lambda_{n}\right| M_{1}+\left|s_{n+1}-s_{n}\right|\left\|B u_{n}\right\| .
\end{aligned}
$$

Hence, we have

$$
\begin{aligned}
\left\|z_{n+1}-z_{n}\right\| \leq & \left\|P_{C}\left(y_{n+1}-r_{n+1} A y_{n+1}\right)-P_{C}\left(y_{n}-r_{n+1} A y_{n}\right)\right\| \\
& +\left\|P_{C}\left(y_{n}-r_{n+1} A y_{n}\right)-P_{C}\left(y_{n}-r_{n} A y_{n}\right)\right\| \\
\leq & \left\|y_{n+1}-y_{n}\right\|+\left|r_{n+1}-r_{n}\right|\left\|A y_{n}\right\| \\
\leq & \left\|x_{n+1}-x_{n}\right\|+M_{2}\left(\left|\lambda_{n+1}-\lambda_{n}\right|+\left|s_{n+1}-s_{n}\right|+\left|r_{n+1}-r_{n}\right|\right),
\end{aligned}
$$

where $M_{2}=\max \left\{M_{1}, \sup _{n \geq 1}\left\{A y_{n}\right\}, \sup _{n \geq 1}\left\{B u_{n}\right\}\right\}$. This implies from (2.5) that

$$
\begin{aligned}
&\left\|W_{n+1} z_{n+1}-W_{n} z_{n}\right\| \\
& \leq\left\|W_{n+1} z_{n+1}-W z_{n+1}\right\|+\left\|W z_{n+1}-W z_{n}\right\|+\left\|W z_{n}-W_{n} z_{n}\right\| \\
& \leq \sup _{x \in K}\left\{\left\|W_{n+1} x-W x\right\|+\left\|W x-W_{n} x\right\|\right\}+\left\|x_{n+1}-x_{n}\right\| \\
&+M_{2}\left(\left|\lambda_{n+1}-\lambda_{n}\right|+\left|s_{n+1}-s_{n}\right|+\left|r_{n+1}-r_{n}\right|\right),
\end{aligned}
$$

where $K$ is the bounded subset of $C$ defined above. Let $x_{n+1}=\left(1-\gamma_{n}\right) q_{n}+\gamma_{n} x_{n}$. It follows that

$$
\begin{aligned}
q_{n+1}-q_{n}= & \frac{\alpha_{n+1} f\left(y_{n+1}\right)+\beta_{n+1} W_{n+1} z_{n+1}}{1-\gamma_{n+1}}-\frac{\alpha_{n} f\left(y_{n}\right)+\beta_{n} W_{n} z_{n}}{1-\gamma_{n}} \\
= & \frac{\alpha_{n+1}}{1-\gamma_{n+1}} f\left(y_{n+1}\right)+\frac{1-\alpha_{n+1}-\gamma_{n+1}}{1-\gamma_{n+1}} W_{n+1} z_{n+1} \\
& -\left(\frac{\alpha_{n}}{1-\gamma_{n}} f\left(y_{n}\right)+\frac{1-\alpha_{n}-\gamma_{n}}{1-\gamma_{n}} W_{n} z_{n}\right) \\
= & \frac{\alpha_{n+1}}{1-\gamma_{n+1}}\left(f\left(y_{n+1}\right)-W_{n+1} z_{n+1}\right)-\frac{\alpha_{n}}{1-\gamma_{n}}\left(f\left(y_{n}\right)-W_{n} z_{n}\right) \\
& +W_{n+1} z_{n+1}-W_{n} z_{n} .
\end{aligned}
$$


By use of (2.6), we find that

$$
\begin{aligned}
\left\|q_{n+1}-q_{n}\right\| \leq & \frac{\alpha_{n+1}}{1-\gamma_{n+1}}\left\|f\left(y_{n+1}\right)-W_{n+1} z_{n+1}\right\|+\frac{\alpha_{n}}{1-\gamma_{n}}\left\|f\left(y_{n}\right)-W_{n} z_{n}\right\| \\
& +\left\|W_{n+1} z_{n+1}-W_{n} z_{n}\right\| \\
\leq & \frac{\alpha_{n+1}}{1-\gamma_{n+1}}\left\|f\left(y_{n+1}\right)-W_{n+1} z_{n+1}\right\|+\frac{\alpha_{n}}{1-\gamma_{n}}\left\|f\left(y_{n}\right)-W_{n} z_{n}\right\| \\
& +\sup _{x \in K}\left\{\left\|W_{n+1} x-W x\right\|+\left\|W x-W_{n} x\right\|\right\}+\left\|x_{n+1}-x_{n}\right\| \\
& +M_{2}\left(\left|\lambda_{n+1}-\lambda_{n}\right|+\left|s_{n+1}-s_{n}\right|+\left|r_{n+1}-r_{n}\right|\right) .
\end{aligned}
$$

This implies that

$$
\begin{aligned}
& \left\|q_{n+1}-q_{n}\right\|-\left\|x_{n+1}-x_{n}\right\| \\
& \leq \frac{\alpha_{n+1}}{1-\gamma_{n+1}}\left\|f\left(y_{n+1}\right)-W_{n+1} z_{n+1}\right\|+\frac{\alpha_{n}}{1-\gamma_{n}}\left\|f\left(y_{n}\right)-W_{n} z_{n}\right\| \\
& \quad+\sup _{x \in K}\left\{\left\|W_{n+1} x-W x\right\|+\left\|W x-W_{n} x\right\|\right\} \\
& \quad+M_{2}\left(\left|\lambda_{n+1}-\lambda_{n}\right|+\left|s_{n+1}-s_{n}\right|+\left|r_{n+1}-r_{n}\right|\right) .
\end{aligned}
$$

It follows from restrictions (a)-(e) that

$$
\limsup _{n \rightarrow \infty}\left(\left\|q_{n+1}-q_{n}\right\|-\left\|x_{n+1}-x_{n}\right\|\right) \leq 0 .
$$

This implies from Lemma 1.4 that $\lim _{n \rightarrow \infty}\left\|q_{n}-x_{n}\right\|=0$. It follows that

$$
\lim _{n \rightarrow \infty}\left\|x_{n+1}-x_{n}\right\|=0
$$

Since $A$ is inverse-strongly monotone, we find that

$$
\begin{aligned}
\left\|z_{n}-x^{*}\right\|^{2} & \leq\left\|\left(I-r_{n} A\right) y_{n}-\left(I-r_{n} A\right) x^{*}\right\|^{2} \\
& \leq\left\|y_{n}-x^{*}\right\|^{2}-2 r_{n} \alpha\left\|A y_{n}-A x^{*}\right\|^{2}+r_{n}^{2}\left\|A y_{n}-A x^{*}\right\|^{2} \\
& \leq\left\|x_{n}-x^{*}\right\|^{2}+r_{n}\left(r_{n}-2 \alpha\right)\left\|A y_{n}-A x^{*}\right\|^{2} .
\end{aligned}
$$

It follows that

$$
\begin{aligned}
\left\|x_{n+1}-x^{*}\right\|^{2} & \leq \alpha_{n}\left\|f\left(y_{n}\right)-x^{*}\right\|^{2}+\beta_{n}\left\|W_{n} z_{n}-x^{*}\right\|^{2}+\gamma_{n}\left\|x_{n}-x^{*}\right\|^{2} \\
& \leq \alpha_{n}\left\|f\left(y_{n}\right)-x^{*}\right\|^{2}+\beta_{n}\left\|z_{n}-x^{*}\right\|^{2}+\gamma_{n}\left\|x_{n}-x^{*}\right\|^{2} \\
& \leq \alpha_{n}\left\|f\left(y_{n}\right)-x^{*}\right\|^{2}+r_{n}\left(r_{n}-2 \alpha\right) \beta_{n}\left\|A y_{n}-A x^{*}\right\|^{2}+\left\|x_{n}-x^{*}\right\|^{2} .
\end{aligned}
$$

Hence, we have

$$
\begin{aligned}
& r_{n}\left(2 \alpha-r_{n}\right) \beta_{n}\left\|A y_{n}-A x^{*}\right\|^{2} \\
& \quad \leq \alpha_{n}\left\|f\left(y_{n}\right)-x^{*}\right\|^{2}+\left(\left\|x_{n}-x^{*}\right\|+\left\|x_{n+1}-x^{*}\right\|\right)\left\|x_{n}-x_{n+1}\right\| .
\end{aligned}
$$


By use of the restrictions (a), (d), and (e), we obtain from (2.7)

$$
\lim _{n \rightarrow \infty}\left\|A y_{n}-A x^{*}\right\|=0 .
$$

Since the metric projection is firmly nonexpansive, we find that

$$
\begin{aligned}
\left\|z_{n}-x^{*}\right\|^{2} \leq & \left\{\left(I-r_{n} A\right) y_{n}-\left(I-r_{n} A\right) x^{*}, z_{n}-x^{*}\right\rangle \\
= & \frac{1}{2}\left\{\left\|\left(I-r_{n} A\right) y_{n}-\left(I-r_{n} A\right) x^{*}\right\|^{2}+\left\|z_{n}-x^{*}\right\|^{2}\right. \\
& \left.-\left\|\left(I-r_{n} A\right) y_{n}-\left(I-r_{n} A\right) x^{*}-\left(z_{n}-x^{*}\right)\right\|^{2}\right\} \\
\leq & \frac{1}{2}\left\{\left\|y_{n}-x^{*}\right\|^{2}+\left\|z_{n}-x^{*}\right\|^{2}-\left\|y_{n}-z_{n}-r_{n}\left(A y_{n}-A x^{*}\right)\right\|^{2}\right\} \\
\leq & \frac{1}{2}\left\{\left\|x_{n}-x^{*}\right\|^{2}+\left\|z_{n}-x^{*}\right\|^{2}-\left\|y_{n}-z_{n}\right\|^{2}\right. \\
& \left.+2 r_{n}\left\|y_{n}-z_{n}\right\|\left\|A y_{n}-A x^{*}\right\|\right\} .
\end{aligned}
$$

Hence, we have

$$
\left\|z_{n}-x^{*}\right\|^{2} \leq\left\|x_{n}-x^{*}\right\|^{2}-\left\|y_{n}-z_{n}\right\|^{2}+2 r_{n}\left\|y_{n}-z_{n}\right\|\left\|A y_{n}-A x^{*}\right\| .
$$

This further implies that

$$
\begin{aligned}
\left\|x_{n+1}-x^{*}\right\|^{2} \leq & \alpha_{n}\left\|f\left(y_{n}\right)-x^{*}\right\|^{2}+\beta_{n}\left\|W_{n} z_{n}-x^{*}\right\|^{2}+\gamma_{n}\left\|x_{n}-x^{*}\right\|^{2} \\
\leq & \alpha_{n}\left\|f\left(y_{n}\right)-x^{*}\right\|^{2}+\beta_{n}\left\|z_{n}-x^{*}\right\|^{2}+\gamma_{n}\left\|x_{n}-x^{*}\right\|^{2} \\
\leq & \alpha_{n}\left\|f\left(y_{n}\right)-x^{*}\right\|^{2}-\beta_{n}\left\|y_{n}-z_{n}\right\|^{2}+2 r_{n}\left\|y_{n}-z_{n}\right\|\left\|A y_{n}-A x^{*}\right\| \\
& \quad+\left\|x_{n}-x^{*}\right\|^{2},
\end{aligned}
$$

which yields

$$
\begin{aligned}
\beta_{n}\left\|y_{n}-z_{n}\right\|^{2} \leq & \alpha_{n}\left\|f\left(y_{n}\right)-x^{*}\right\|^{2}+2 r_{n}\left\|y_{n}-z_{n}\right\|\left\|A y_{n}-A x^{*}\right\| \\
& +\left\|x_{n}-x^{*}\right\|^{2}-\left\|x_{n+1}-x^{*}\right\|^{2} \\
\leq & \alpha_{n}\left\|f\left(y_{n}\right)-x^{*}\right\|^{2}+2 r_{n}\left\|y_{n}-z_{n}\right\|\left\|A y_{n}-A x^{*}\right\| \\
& +\left(\left\|x_{n}-x^{*}\right\|+\left\|x_{n+1}-x^{*}\right\|\right)\left\|x_{n+1}-x_{n}\right\| .
\end{aligned}
$$

By use of restrictions (b), (d), and (e), we find from (2.7) that

$$
\lim _{n \rightarrow \infty}\left\|y_{n}-z_{n}\right\|=0
$$

Since $B$ is inverse-strongly monotone, we find that

$$
\begin{aligned}
\left\|y_{n}-x^{*}\right\|^{2} & \leq\left\|\left(I-s_{n} B\right) u_{n}-\left(I-s_{n} B\right) x^{*}\right\|^{2} \\
& \leq\left\|u_{n}-x^{*}\right\|^{2}-2 s_{n} \beta\left\|B u_{n}-B x^{*}\right\|^{2}+s_{n}^{2}\left\|B u_{n}-B x^{*}\right\|^{2} \\
& \leq\left\|x_{n}-x^{*}\right\|^{2}+s_{n}\left(s_{n}-2 \beta\right)\left\|B u_{n}-B x^{*}\right\|^{2} .
\end{aligned}
$$


It follows that

$$
\begin{aligned}
\left\|x_{n+1}-x^{*}\right\|^{2} & \leq \alpha_{n}\left\|f\left(y_{n}\right)-x^{*}\right\|^{2}+\beta_{n}\left\|W_{n} z_{n}-x^{*}\right\|^{2}+\gamma_{n}\left\|x_{n}-x^{*}\right\|^{2} \\
& \leq \alpha_{n}\left\|f\left(y_{n}\right)-x^{*}\right\|^{2}+\beta_{n}\left\|y_{n}-x^{*}\right\|^{2}+\gamma_{n}\left\|x_{n}-x^{*}\right\|^{2} \\
& \leq \alpha_{n}\left\|f\left(y_{n}\right)-x^{*}\right\|^{2}+s_{n}\left(s_{n}-2 \beta\right) \beta_{n}\left\|B u_{n}-B x^{*}\right\|^{2}+\left\|x_{n}-x^{*}\right\|^{2} .
\end{aligned}
$$

Hence, we have

$$
\begin{aligned}
& s_{n}\left(2 \beta-s_{n}\right) \beta_{n}\left\|B u_{n}-B x^{*}\right\|^{2} \\
& \quad \leq \alpha_{n}\left\|f\left(y_{n}\right)-x^{*}\right\|^{2}+\left(\left\|x_{n}-x^{*}\right\|+\left\|x_{n+1}-x^{*}\right\|\right)\left\|x_{n}-x_{n+1}\right\| .
\end{aligned}
$$

By use of the restrictions (c), (d), and (e), we obtain from (2.7)

$$
\lim _{n \rightarrow \infty}\left\|B u_{n}-B x^{*}\right\|=0
$$

Since the metric projection is firmly nonexpansive, we find that

$$
\begin{aligned}
\left\|y_{n}-x^{*}\right\|^{2} \leq & \left\{\left(I-s_{n} B\right) u_{n}-\left(I-s_{n} B\right) x^{*}, y_{n}-x^{*}\right\rangle \\
= & \frac{1}{2}\left\{\left\|\left(I-s_{n} B\right) u_{n}-\left(I-s_{n} B\right) x^{*}\right\|^{2}+\left\|y_{n}-x^{*}\right\|^{2}\right. \\
& \left.-\left\|\left(I-s_{n} B\right) y_{n}-\left(I-s_{n} B\right) x^{*}-\left(y_{n}-x^{*}\right)\right\|^{2}\right\} \\
\leq & \frac{1}{2}\left\{\left\|u_{n}-x^{*}\right\|^{2}+\left\|y_{n}-x^{*}\right\|^{2}-\left\|u_{n}-y_{n}-s_{n}\left(B u_{n}-B x^{*}\right)\right\|^{2}\right\} \\
\leq & \frac{1}{2}\left\{\left\|x_{n}-x^{*}\right\|^{2}+\left\|y_{n}-x^{*}\right\|^{2}-\left\|u_{n}-y_{n}\right\|^{2}\right. \\
& \left.+2 s_{n}\left\|u_{n}-y_{n}\right\|\left\|B u_{n}-B x^{*}\right\|\right\} .
\end{aligned}
$$

Hence, we have

$$
\left\|y_{n}-x^{*}\right\|^{2} \leq\left\|x_{n}-x^{*}\right\|^{2}-\left\|u_{n}-y_{n}\right\|^{2}+2 s_{n}\left\|u_{n}-y_{n}\right\|\left\|B u_{n}-B x^{*}\right\| .
$$

This further implies that

$$
\begin{aligned}
\left\|x_{n+1}-x^{*}\right\|^{2} \leq & \alpha_{n}\left\|f\left(y_{n}\right)-x^{*}\right\|^{2}+\beta_{n}\left\|W_{n} z_{n}-x^{*}\right\|^{2}+\gamma_{n}\left\|x_{n}-x^{*}\right\|^{2} \\
\leq & \alpha_{n}\left\|f\left(y_{n}\right)-x^{*}\right\|^{2}+\beta_{n}\left\|y_{n}-x^{*}\right\|^{2}+\gamma_{n}\left\|x_{n}-x^{*}\right\|^{2} \\
\leq & \alpha_{n}\left\|f\left(y_{n}\right)-x^{*}\right\|^{2}-\beta_{n}\left\|u_{n}-y_{n}\right\|^{2}+2 s_{n}\left\|u_{n}-y_{n}\right\|\left\|B u_{n}-B x^{*}\right\| \\
& +\left\|x_{n}-x^{*}\right\|^{2},
\end{aligned}
$$


which yields

$$
\begin{aligned}
\beta_{n}\left\|u_{n}-y_{n}\right\|^{2} \leq & \alpha_{n}\left\|f\left(y_{n}\right)-x^{*}\right\|^{2}+2 s_{n}\left\|u_{n}-y_{n}\right\|\left\|B u_{n}-B x^{*}\right\| \\
& +\left\|x_{n}-x^{*}\right\|^{2}-\left\|x_{n+1}-x^{*}\right\|^{2} \\
\leq & \alpha_{n}\left\|f\left(y_{n}\right)-x^{*}\right\|^{2}+2 s_{n}\left\|u_{n}-y_{n}\right\|\left\|B u_{n}-B x^{*}\right\| \\
& +\left(\left\|x_{n}-x^{*}\right\|+\left\|x_{n+1}-x^{*}\right\|\right)\left\|x_{n+1}-x_{n}\right\| .
\end{aligned}
$$

By use of restrictions (c), (d), and (e), we find from (2.7) that

$$
\lim _{n \rightarrow \infty}\left\|y_{n}-u_{n}\right\|=0
$$

Since $T$ is inverse-strongly monotone, we find that

$$
\begin{aligned}
\left\|x_{n+1}-x^{*}\right\|^{2} & \leq \alpha_{n}\left\|f\left(y_{n}\right)-x^{*}\right\|^{2}+\beta_{n}\left\|u_{n}-x^{*}\right\|^{2}+\gamma_{n}\left\|x_{n}-x^{*}\right\|^{2} \\
& \leq \alpha_{n}\left\|f\left(y_{n}\right)-x^{*}\right\|^{2}+\beta_{n}\left\|x_{n}-x^{*}-\lambda_{n}\left(T x_{n}-T x^{*}\right)\right\|^{2}+\gamma_{n}\left\|x_{n}-x^{*}\right\|^{2} \\
& \leq \alpha_{n}\left\|f\left(y_{n}\right)-x^{*}\right\|^{2}+\left\|x_{n}-x^{*}\right\|^{2}-\lambda_{n} \beta_{n}\left(2 \tau-\lambda_{n}\right)\left\|T x_{n}-T x^{*}\right\|^{2} .
\end{aligned}
$$

This implies that

$$
\begin{aligned}
& \lambda_{n} \beta_{n}\left(2 \tau-\lambda_{n}\right)\left\|T x_{n}-T x^{*}\right\|^{2} \\
& \quad \leq \alpha_{n}\left\|f\left(y_{n}\right)-x^{*}\right\|^{2}+\left(\left\|x_{n}-x^{*}\right\|+\left\|x_{n+1}-x^{*}\right\|\right)\left\|x_{n}-x_{n+1}\right\| .
\end{aligned}
$$

In view of the restrictions (a), (d), and (e), we see from (2.7) that

$$
\lim _{n \rightarrow \infty}\left\|T x_{n}-T x^{*}\right\|=0 .
$$

Since $T_{\lambda_{n}}$ is firmly nonexpansive, we find that

$$
\begin{aligned}
\left\|u_{n}-x^{*}\right\|^{2}= & \left\|T_{\lambda_{n}}\left(I-\lambda_{n} T\right) x_{n}-T_{\lambda_{n}}\left(I-\lambda_{n} T\right) x^{*}\right\|^{2} \\
\leq & \left\{\left(I-\lambda_{n} T\right) x_{n}-\left(I-\lambda_{n} T\right) x^{*}, u_{n}-x^{*}\right\rangle \\
\leq & \frac{1}{2}\left(\left\|x_{n}-x^{*}\right\|^{2}+\left\|u_{n}-x^{*}\right\|^{2}-\left\|x_{n}-u_{n}\right\|^{2}\right. \\
& \left.+2 \lambda_{n}\left\|T x_{n}-T x^{*}\right\|\left\|x_{n}-u_{n}\right\|\right) .
\end{aligned}
$$

This in turn implies that

$$
\left\|u_{n}-x^{*}\right\|^{2} \leq\left\|x_{n}-x^{*}\right\|^{2}-\left\|x_{n}-u_{n}\right\|^{2}+2 \lambda_{n}\left\|T x_{n}-T x^{*}\right\|\left\|x_{n}-u_{n}\right\| .
$$

It follows that

$$
\begin{aligned}
\left\|x_{n+1}-x^{*}\right\|^{2} \leq & \alpha_{n}\left\|f\left(y_{n}\right)-x^{*}\right\|^{2}+\beta_{n}\left\|u_{n}-x^{*}\right\|^{2}+\gamma_{n}\left\|x_{n}-x^{*}\right\|^{2} \\
\leq & \alpha_{n}\left\|f\left(y_{n}\right)-x^{*}\right\|^{2}-\beta_{n}\left\|x_{n}-u_{n}\right\|^{2} \\
& +2 \lambda_{n}\left\|T x_{n}-T x^{*}\right\|\left\|x_{n}-u_{n}\right\|+\left\|x_{n}-x^{*}\right\|^{2} .
\end{aligned}
$$


By use of restrictions (a), (d), and (e), we see from (2.7) and (2.12) that

$$
\lim _{n \rightarrow \infty}\left\|x_{n}-u_{n}\right\|=0
$$

Next, we prove that

$$
\limsup _{n \rightarrow \infty}\left\langle f(\bar{x})-\bar{x}, x_{n}-\bar{x}\right\rangle \leq 0
$$

where $\bar{x}=P_{\Sigma} f(\bar{x})$. To see this, we choose a subsequence $\left\{x_{n_{i}}\right\}$ of $\left\{x_{n}\right\}$ such that

$$
\limsup _{n \rightarrow \infty}\left\langle(f-I) \bar{x}, x_{n}-\bar{x}\right\rangle=\lim _{i \rightarrow \infty}\left\langle(f-I) \bar{x}, x_{n_{i}}-\bar{x}\right\rangle .
$$

Since $\left\{x_{n_{i}}\right\}$ is bounded, there exists a subsequence $\left\{x_{n_{i_{j}}}\right\}$ of $\left\{x_{n_{i}}\right\}$ which converges weakly to $w$. Without loss of generality, we may assume that $x_{n_{i}} \rightarrow w$. Since

$$
\beta_{n}\left\|W_{n} z_{n}-x_{n}\right\| \leq\left\|x_{n}-x_{n+1}\right\|+\alpha_{n}\left\|f\left(y_{n}\right)-x_{n}\right\|
$$

In view of the restrictions (d) and (e), we obtain from (2.7)

$$
\lim _{n \rightarrow \infty}\left\|W_{n} z_{n}-x_{n}\right\|=0
$$

Note that

$$
\left\|W_{n} z_{n}-z_{n}\right\| \leq\left\|W_{n} z_{n}-x_{n}\right\|+\left\|x_{n}-u_{n}\right\|+\left\|u_{n}-y_{n}\right\|+\left\|y_{n}-z_{n}\right\| .
$$

In view of (2.8), (2.11), (2.13), and (2.14), we find that

$$
\lim _{n \rightarrow \infty}\left\|W_{n} z_{n}-z_{n}\right\|=0
$$

Suppose the contrary, $w \notin \bigcap_{i=1}^{\infty} F\left(S_{i}\right)$, i.e., $W w \neq w$. Since $y_{n_{i}} \rightarrow w$, we find from Opial's condition [32] that

$$
\begin{aligned}
\liminf _{i \rightarrow \infty}\left\|z_{n_{i}}-w\right\| & <\liminf _{i \rightarrow \infty}\left\|z_{n_{i}}-W w\right\| \\
& \leq \liminf _{i \rightarrow \infty}\left\{\left\|z_{n_{i}}-W z_{n_{i}}\right\|+\left\|W z_{n_{i}}-W w\right\|\right\} \\
& \leq \liminf _{i \rightarrow \infty}\left\{\left\|z_{n_{i}}-W z_{n_{i}}\right\|+\left\|z_{n_{i}}-w\right\|\right\} .
\end{aligned}
$$

On the other hand, we have

$$
\begin{aligned}
\left\|W z_{n}-z_{n}\right\| & \leq\left\|W z_{n}-W_{n} z_{n}\right\|+\left\|W_{n} z_{n}-y_{n}\right\| \\
& \leq \sup _{x \in K}\left\|W x-W_{n} x\right\|+\left\|W_{n} z_{n}-z_{n}\right\| .
\end{aligned}
$$

In view of Lemma 1.5, we obtain from (2.15) $\lim _{n \rightarrow \infty}\left\|W z_{n}-z_{n}\right\|=0$. It follows that $\liminf _{i \rightarrow \infty}\left\|z_{n_{i}}-w\right\|<\liminf _{i \rightarrow \infty}\left\|z_{n_{i}}-w\right\|$. Thus one derives a contradiction. Thus, we have $w \in \bigcap_{i=1}^{\infty} F\left(S_{i}\right)$. 
Next, we show that $\bar{x} \in V I(C, A)$. Let $T$ be the maximal monotone mapping defined by

$$
D x= \begin{cases}B x+N_{C} x, & x \in C, \\ \emptyset, & x \notin C .\end{cases}
$$

For any given $(x, y) \in \operatorname{Graph}(D)$, we have $y-B x \in N_{C} x$. Since $y_{n} \in C$, by the definition of $N_{C}$, we have $\left\langle x-y_{n}, y-B x\right\rangle \geq 0$. Since $y_{n}=P_{C}\left(u_{n}-s_{n} B u_{n}\right)$, we see that $\left\langle x-y_{n}, \frac{y_{n}-u_{n}}{s_{n}}+B u_{n}\right\rangle \geq 0$. It follows that

$$
\begin{aligned}
\left\langle x-y_{n_{i}}, y\right\rangle & \geq\left\langle x-y_{n_{i}}, B x\right\rangle \\
& \geq\left\langle x-y_{n_{i}}, B x\right\rangle-\left\langle x-y_{n_{i}}, \frac{y_{n_{i}}-u_{n_{i}}}{s_{n_{i}}}+B u_{n_{i}}\right\rangle \\
& =\left\langle x-y_{n_{i}}, B x-B y_{n_{i}}\right\rangle+\left\langle x-y_{n_{i}}, B y_{n_{i}}-B u_{n_{i}}\right\rangle-\left\langle x-y_{n_{i}}, \frac{y_{n_{i}}-u_{n_{i}}}{s_{n_{i}}}\right\rangle \\
& \geq\left\langle x-y_{n_{i}}, B y_{n_{i}}-B u_{n_{i}}\right\rangle-\left\langle x-y_{n_{i}}, \frac{y_{n_{i}}-u_{n_{i}}}{s_{n_{i}}}\right\rangle .
\end{aligned}
$$

Since $B$ is Lipschitz continuous, we see that $\langle x-w, y\rangle \geq 0$. Notice that $D$ is maximal monotone and hence $0 \in T w$. This shows that $w \in V I(C, A)$. In the same way, we find that $w \in V I(C, B)$.

Next, we show that $w \in \operatorname{GEP}(F, T)$. Since $u_{n}=T_{\lambda_{n}}\left(I-\lambda_{n} T\right) x_{n}$, for any $y \in C$, we find from (A2) that

$$
\left\langle T x_{n_{i}}, y-u_{n_{i}}\right\rangle+\left\langle y-u_{n_{i}}, \frac{u_{n_{i}}-x_{n_{i}}}{\lambda_{n_{i}}}\right\rangle \geq F\left(y, u_{n_{i}}\right), \quad \forall y \in C .
$$

Putting $y_{t}=t y+(1-t) w$ for any $t \in(0,1]$ and $y \in C$, we see that $y_{t} \in C$. It follows from (2.16) that

$$
\begin{aligned}
& \left\langle y_{t}-u_{n_{i}}, T y_{t}\right\rangle \\
& \quad \geq\left\langle y_{t}-u_{n_{i}}, T y_{t}\right\rangle-\left\langle T x_{n_{i}}, y_{t}-u_{n_{i}}\right\rangle-\left\langle y_{t}-u_{n_{i}}, \frac{u_{n_{i}}-x_{n_{i}}}{\lambda_{n_{i}}}\right\rangle+F\left(y_{t}, u_{n_{i}}\right) \\
& =\left\langle y_{t}-u_{n_{i}}, T y_{t}-T u_{n_{i}}\right\rangle+\left\langle y_{t}-u_{n_{i}}, T u_{n_{i}}-T x_{n_{i}}\right\rangle-\left\langle y_{t}-u_{n_{i}}, \frac{u_{n_{i}}-x_{n_{i}}}{\lambda_{n_{i}}}\right\rangle+F\left(y_{t}, u_{n_{i}}\right) .
\end{aligned}
$$

In view of the monotonicity of $T$, and the restriction (a), we obtain from (A4)

$$
\left\langle y_{t}-w, T y_{t}\right\rangle \geq F\left(y_{t}, q\right)
$$

From (A1) and (A4), we see that

$$
\begin{aligned}
0 & =F\left(y_{t}, y_{t}\right) \leq t F\left(y_{t}, y\right)+(1-t) F\left(y_{t}, w\right) \\
& \leq t F\left(y_{t}, y\right)+(1-t)\left\langle y_{t}-w, T y_{t}\right\rangle \\
& =t F\left(y_{t}, y\right)+(1-t) t\left\langle y-w, T y_{t}\right\rangle .
\end{aligned}
$$

It follows from (A3) that $w \in \operatorname{GEP}(F, T)$. This proves that $\limsup _{n \rightarrow \infty}\left\langle f(\bar{x})-\bar{x}, x_{n}-\bar{x}\right\rangle \leq 0$. 
Finally, we show that $x_{n} \rightarrow \bar{x}$, as $n \rightarrow \infty$. Note that

$$
\begin{aligned}
&\left\|x_{n+1}-\bar{x}\right\|^{2} \\
&= \alpha_{n}\left\langle f\left(y_{n}\right)-\bar{x}, x_{n+1}-\bar{x}\right\rangle+\beta_{n}\left\langle W_{n} z_{n}-\bar{x}, x_{n+1}-\bar{x}\right\rangle+\gamma_{n}\left\langle x_{n}-\bar{x}, x_{n+1}-\bar{x}\right\rangle \\
& \leq \alpha_{n} \kappa\left\|y_{n}-\bar{x}\right\|\left\|x_{n+1}-\bar{x}\right\|+\alpha_{n}\left\langle f(\bar{x})-\bar{x}, x_{n+1}-\bar{x}\right\rangle+\beta_{n}\left\|z_{n}-\bar{x}\right\|\left\|x_{n+1}-\bar{x}\right\| \\
&+\gamma_{n}\left\|x_{n}-\bar{x}\right\|\left\|x_{n+1}-\bar{x}\right\| \\
& \leq\left(1-\alpha_{n}(1-\kappa)\right)\left\|x_{n}-\bar{x}\right\|\left\|x_{n+1}-\bar{x}\right\|+\alpha_{n}\left\langle f(\bar{x})-\bar{x}, x_{n+1}-\bar{x}\right\rangle .
\end{aligned}
$$

This implies that

$$
\left\|x_{n+1}-\bar{x}\right\|^{2} \leq\left(1-\alpha_{n}(1-\kappa)\right)\left\|x_{n}-\bar{x}\right\|^{2}+2 \alpha_{n}\left\langle f(\bar{x})-\bar{x}, x_{n+1}-\bar{x}\right\rangle .
$$

From the restriction (d), we obtain from Lemma $1.2 \lim _{n \rightarrow \infty}\left\|x_{n}-\bar{x}\right\|=0$. This completes the proof.

Corollary 2.2 Let $C$ be a nonempty closed convex subset of $H$. Let $F$ be a bifunction from $C \times C$ to $\mathbb{R}$ which satisfies (A1)-(A4) and let $f: C \rightarrow C$ be a $\kappa$-contraction. Let $T: C \rightarrow$ $H$ be a $\tau$-inverse-strongly monotone mapping. Let $\left\{S_{i}: C \rightarrow C\right\}$ be a family of infinitely nonexpansive mappings. Assume that $\Sigma=\bigcap_{i=1}^{\infty} F\left(S_{i}\right) \cap \operatorname{GEP}(F, T)$ is not empty. Let $\left\{\alpha_{n}\right\}$, $\left\{\beta_{n}\right\}$, and $\left\{\gamma_{n}\right\}$ be sequences in $(0,1)$ such that $\alpha_{n}+\beta_{n}+\gamma_{n}=1$. Let $\left\{\lambda_{n}\right\}$ be a positive number sequence. Let $x_{1} \in C$ and let $\left\{x_{n}\right\}$ be a sequence generated by

$$
x_{n+1}=\alpha_{n} f\left(y_{n}\right)+\beta_{n} W_{n} u_{n}+\gamma_{n} x_{n}, \quad \forall n \geq 1,
$$

where $\left\{u_{n}\right\}$ is such that $F\left(u_{n}, y\right)+\left\langle T x_{n}, y-u_{n}\right\rangle+\frac{1}{\lambda_{n}}\left\langle y-u_{n}, u_{n}-x_{n}\right\rangle \geq 0, \forall y \in C$, and $\left\{W_{n}\right\}$ is the sequence generated in (1.5). Assume that the following restrictions hold:

(a) $0<a \leq \lambda_{n} \leq b<2 \tau$ and $\lim _{n \rightarrow \infty}\left|\lambda_{n+1}-\lambda_{n}\right|=0$,

(b) $\lim _{n \rightarrow \infty} \alpha_{n}=0, \sum_{n=1}^{\infty} \alpha_{n}=\infty$,

(c) $0<\liminf _{n \rightarrow \infty} \gamma_{n} \leq \limsup _{n \rightarrow \infty} \gamma_{n}<1$,

where $a$ and $b$ are real constants. Then $\left\{x_{n}\right\}$ converges strongly to $\bar{x} \in \Sigma$, which solves uniquely the following variational inequality:

$$
\langle\bar{x}-f(\bar{x}), \bar{x}-x\rangle \leq 0, \quad \forall x \in \Sigma .
$$

Corollary 2.3 Let $C$ be a nonempty closed convex subset of $H$. Let $F$ be a bifunction from $C \times C$ to $\mathbb{R}$ which satisfies (A1)-(A4) and let $f: C \rightarrow C$ be a $\kappa$-contraction. Let $B: C \rightarrow H$ be a $\beta$-inverse-strongly monotone mapping. Let $T: C \rightarrow H$ be a $\tau$-inverse-strongly monotone mapping. Let $\left\{S_{i}: C \rightarrow C\right\}$ be a family of infinitely nonexpansive mappings. Assume that $\Sigma=\bigcap_{i=1}^{\infty} F\left(S_{i}\right) \cap \operatorname{GEP}(F, T) \cap V I(C, B)$ is not empty. Let $\left\{\alpha_{n}\right\},\left\{\beta_{n}\right\}$, and $\left\{\gamma_{n}\right\}$ be sequences in $(0,1)$ such that $\alpha_{n}+\beta_{n}+\gamma_{n}=1$. Let $\left\{s_{n}\right\}$ and $\left\{\lambda_{n}\right\}$ be positive number sequences. Let $x_{1} \in C$ and let $\left\{x_{n}\right\}$ be a sequence generated by

$$
\left\{\begin{array}{l}
y_{n}=P_{C}\left(u_{n}-s_{n} B u_{n}\right), \\
x_{n+1}=\alpha_{n} f\left(y_{n}\right)+\beta_{n} W_{n} y_{n}+\gamma_{n} x_{n}, \quad \forall n \geq 1,
\end{array}\right.
$$


where $\left\{u_{n}\right\}$ is such that $F\left(u_{n}, y\right)+\left\langle T x_{n}, y-u_{n}\right\rangle+\frac{1}{\lambda_{n}}\left\langle y-u_{n}, u_{n}-x_{n}\right\rangle \geq 0, \forall y \in C$, and $\left\{W_{n}\right\}$ is the sequence generated in (1.5). Assume that the following restrictions hold:

(a) $0<a \leq \lambda_{n} \leq b<2 \tau$ and $\lim _{n \rightarrow \infty}\left|\lambda_{n+1}-\lambda_{n}\right|=0$,

(b) $0<a^{\prime \prime} \leq s_{n} \leq b^{\prime \prime}<2 \beta$ and $\lim _{n \rightarrow \infty}\left|s_{n+1}-s_{n}\right|=0$,

(c) $\lim _{n \rightarrow \infty} \alpha_{n}=0, \sum_{n=1}^{\infty} \alpha_{n}=\infty$,

(d) $0<\liminf _{n \rightarrow \infty} \gamma_{n} \leq \limsup _{n \rightarrow \infty} \gamma_{n}<1$,

where $a, a^{\prime}, b$, and $b^{\prime}$ are real constants. Then $\left\{x_{n}\right\}$ converges strongly to $\bar{x} \in \Sigma$, which solves uniquely the following variational inequality:

$$
\langle\bar{x}-f(\bar{x}), \bar{x}-x\rangle \leq 0, \quad \forall x \in \Sigma .
$$

Corollary 2.4 Let $C$ be a nonempty closed convex subset of $H$. Let $F$ be a bifunction from $C \times C$ to $\mathbb{R}$ which satisfies (A1)-(A4) and let $f: C \rightarrow C$ be a $\kappa$-contraction. Let $A: C \rightarrow H$ be an $\alpha$-inverse-strongly monotone mapping and let $B: C \rightarrow H$ be a $\beta$-inverse-strongly monotone mapping. Let $\left\{S_{i}: C \rightarrow C\right\}$ be a family of infinitely nonexpansive mappings. Assume that $\Sigma=\bigcap_{i=1}^{\infty} F\left(S_{i}\right) \cap E P(F) \cap V I(C, A) \cap V I(C, B)$ is not empty. Let $\left\{\alpha_{n}\right\},\left\{\beta_{n}\right\}$, and $\left\{\gamma_{n}\right\}$ be sequences in $(0,1)$ such that $\alpha_{n}+\beta_{n}+\gamma_{n}=1$. Let $\left\{r_{n}\right\}$, $\left\{s_{n}\right\}$, and $\left\{\lambda_{n}\right\}$ be positive number sequences. Let $x_{1} \in C$ and let $\left\{x_{n}\right\}$ be a sequence generated by

$$
\left\{\begin{array}{l}
y_{n}=P_{C}\left(u_{n}-s_{n} B u_{n}\right), \\
x_{n+1}=\alpha_{n} f\left(y_{n}\right)+\beta_{n} W_{n} P_{C}\left(y_{n}-r_{n} A y_{n}\right)+\gamma_{n} x_{n}, \quad \forall n \geq 1,
\end{array}\right.
$$

where $\left\{u_{n}\right\}$ is such that $F\left(u_{n}, y\right)+\frac{1}{\lambda_{n}}\left\langle y-u_{n}, u_{n}-x_{n}\right\rangle \geq 0, \forall y \in C$, and $\left\{W_{n}\right\}$ is the sequence generated in (1.5). Assume that the following restrictions hold:

(a) $0<a \leq \lambda_{n} \leq b<2 \tau$ and $\lim _{n \rightarrow \infty}\left|\lambda_{n+1}-\lambda_{n}\right|=0$,

(b) $0<a^{\prime} \leq r_{n} \leq b^{\prime}<2 \alpha$ and $\lim _{n \rightarrow \infty}\left|r_{n+1}-r_{n}\right|=0$,

(c) $0<a^{\prime \prime} \leq s_{n} \leq b^{\prime \prime}<2 \beta$ and $\lim _{n \rightarrow \infty}\left|s_{n+1}-s_{n}\right|=0$,

(d) $\lim _{n \rightarrow \infty} \alpha_{n}=0, \sum_{n=1}^{\infty} \alpha_{n}=\infty$,

(e) $0<\liminf _{n \rightarrow \infty} \gamma_{n} \leq \limsup _{n \rightarrow \infty} \gamma_{n}<1$,

where $a, a^{\prime}, a^{\prime \prime}, b, b^{\prime}$, and $b^{\prime \prime}$ are real constants. Then $\left\{x_{n}\right\}$ converges strongly to $\bar{x} \in \Sigma$, which solves uniquely the following variational inequality:

$$
\langle\bar{x}-f(\bar{x}), \bar{x}-x\rangle \leq 0, \quad \forall x \in \Sigma .
$$

Proof In Theorem 2.1, put $T=0$. Then, for all $\tau \in(0, \infty)$, we have

$$
\langle x, y, T x-T y\rangle \geq \tau\|T x-T y\|^{2}, \quad \forall x, y \in C .
$$

Taking $a, b \in(0, \infty)$ with $0<a<b<\infty$ and choosing a sequence $\left\{\lambda_{n}\right\}$ of real numbers with $a \leq \lambda_{n} \leq b$, we obtain the desired result by Theorem 2.1.

Corollary 2.5 Let $C$ be a nonempty closed convex subset of $H$. Let $f: C \rightarrow C$ be a $\kappa$ contraction and let $T: C \rightarrow H$ be a $\tau$-inverse-strongly monotone mapping. Let $A: C \rightarrow H$ be an $\alpha$-inverse-strongly monotone mapping and let $B: C \rightarrow H$ be a $\beta$-inverse-strongly monotone mapping. Let $\left\{S_{i}: C \rightarrow C\right\}$ be a family of infinitely nonexpansive mappings. Assume that $\Sigma=\bigcap_{i=1}^{\infty} F\left(S_{i}\right) \cap V I(C, T) \cap V I(C, A) \cap V I(C, B)$ is not empty. Let $\left\{\alpha_{n}\right\},\left\{\beta_{n}\right\}$, and 
$\left\{\gamma_{n}\right\}$ be sequences in $(0,1)$ such that $\alpha_{n}+\beta_{n}+\gamma_{n}=1$. Let $\left\{r_{n}\right\},\left\{s_{n}\right\}$, and $\left\{\lambda_{n}\right\}$ be positive number sequences. Let $x_{1} \in C$ and let $\left\{x_{n}\right\}$ be a sequence generated by

$$
\left\{\begin{array}{l}
u_{n}=P_{C}\left(x_{n}-\lambda_{n} T x_{n}\right), \\
y_{n}=P_{C}\left(u_{n}-s_{n} B u_{n}\right), \\
x_{n+1}=\alpha_{n} f\left(y_{n}\right)+\beta_{n} W_{n} P_{C}\left(y_{n}-r_{n} A y_{n}\right)+\gamma_{n} x_{n}, \quad \forall n \geq 1,
\end{array}\right.
$$

where $\left\{W_{n}\right\}$ is the sequence generated in (1.5). Assume that the following restrictions hold:

(a) $0<a \leq \lambda_{n} \leq b<2 \tau$ and $\lim _{n \rightarrow \infty}\left|\lambda_{n+1}-\lambda_{n}\right|=0$,

(b) $0<a^{\prime} \leq r_{n} \leq b^{\prime}<2 \alpha$ and $\lim _{n \rightarrow \infty}\left|r_{n+1}-r_{n}\right|=0$,

(c) $0<a^{\prime \prime} \leq s_{n} \leq b^{\prime \prime}<2 \beta$ and $\lim _{n \rightarrow \infty}\left|s_{n+1}-s_{n}\right|=0$,

(d) $\lim _{n \rightarrow \infty} \alpha_{n}=0, \sum_{n=1}^{\infty} \alpha_{n}=\infty$,

(e) $0<\liminf _{n \rightarrow \infty} \gamma_{n} \leq \limsup _{n \rightarrow \infty} \gamma_{n}<1$,

where $a, a^{\prime}, a^{\prime \prime}, b, b^{\prime}$, and $b^{\prime \prime}$ are real constants. Then $\left\{x_{n}\right\}$ converges strongly to $\bar{x} \in \Sigma$, which solves uniquely the following variational inequality:

$$
\langle\bar{x}-f(\bar{x}), \bar{x}-x\rangle \leq 0, \quad \forall x \in \Sigma .
$$

Proof Putting $F=0$, we find that

$$
\left\langle T x_{n}, y-u_{n}\right\rangle+\frac{1}{\lambda_{n}}\left\langle y-u_{n}, u_{n}-x_{n}\right\rangle \geq 0, \quad \forall y \in C,
$$

is equivalent to

$$
\left\langle y-u_{n}, x_{n}-\lambda_{n} T x_{n}-u_{n}\right\rangle \leq 0, \quad \forall y \in C,
$$

that is, $u_{n}=P_{C}\left(x_{n}-\lambda_{n} T x_{n}\right)$. This completes the proof.

\section{Competing interests}

The authors declare that they have no competing interests.

\section{Authors' contributions}

Both authors contributed equally to this manuscript. Both authors read and approved the final manuscript.

\section{Author details}

'College of Science, Hangzhou Normal University, Hangzhou, China. ${ }^{2}$ Department of Mathematics, Jinming Institute of Education, Jinming, China.

\section{Acknowledgements}

The authors are grateful to the referees for useful suggestions which improved the contents of the paper.

Received: 28 May 2014 Accepted: 15 August 2014 Published: 2 September 2014

\section{References}

1. Browder, FE: Nonlinear operators and nonlinear equations of evolution in Banach spaces. Proc. Symp. Pure Math. 18, 78-81 (1976)

2. Shimoji, K, Takahashi, W: Strong convergence to common fixed points of infinite nonexpansive mappings and applications. Taiwan. J. Math. 5, 387-404 (2001)

3. Blum, E, Oettli, W: From optimization and variational inequalities to equilibrium problems. Math. Stud. 63, 123-145 (1994)

4. He, RH: Coincidence theorem and existence theorems of solutions for a system of Ky Fan type minimax inequalities in FC-spaces. Adv. Fixed Point Theory 2, 47-57 (2012)

5. Kim, KS, Kim, JK, Lim, WH: Convergence theorems for common solutions of various problems with nonlinear mapping. J. Inequal. Appl. 2014, 2 (2014) 
6. Park, S: A review of the KKM theory on $\phi_{A}$-spaces or GFC-spaces. Adv. Fixed Point Theory 3, 355-382 (2013)

7. Cho, SY, Kang, SM: Approximation of common solutions of variational inequalities via strict pseudocontractions. Acta Math. Sci. 32, 1607-1618 (2012)

8. Zegeye, $\mathrm{H}$, Shahzad, N: Strong convergence theorem for a common point of solution of variational inequality and fixed point problem. Adv. Fixed Point Theory 2, 374-397 (2012)

9. Al-Bayati, AY, Al-Kawaz, RZ: A new hybrid WC-FR conjugate gradient-algorithm with modified secant condition for unconstrained optimization. J. Math. Comput. Sci. 2, 937-966 (2012)

10. Rockafellar, RT: On the maximality of sums of nonlinear monotone operators. Trans. Am. Math. Soc. 149, 75-88 (1970)

11. Chang, SS, Lee, HWJ, Chan, CK: A new method for solving equilibrium problem fixed point problem and variational inequality problem with application to optimization. Nonlinear Anal. 70, 3307-3319 (2009)

12. Qin, X, Chang, SS, Cho, YJ: Iterative methods for generalized equilibrium problems and fixed point problems with applications. Nonlinear Anal. 11, 2963-2972 (2010)

13. Takahashi, S, Takahashi, W: Viscosity approximation methods for equilibrium problems and fixed point problems in Hilbert spaces. J. Math. Anal. Appl. 331, 506-515 (2007)

14. Zhang, L, Tong, $\mathrm{H}$ : An iterative method for nonexpansive semigroups, variational inclusions and generalized equilibrium problems. Adv. Fixed Point Theory 4, 325-343 (2014)

15. Qin, X, Cho, SY, Kang, SM: Iterative algorithms for variational inequality and equilibrium problems with applications. J. Glob. Optim. 48, 423-445 (2010)

16. LV, S: Strong convergence of a general iterative algorithm in Hilbert spaces. J. Inequal. Appl. 2013, Article ID 19 (2013)

17. Li, DF, Zhao, J: On variational inequality, fixed point and generalized mixed equilibrium problems. J. Inequal. Appl. 2014, Article ID 203 (2014)

18. Chen, JH: Iterations for equilibrium and fixed point problems. J. Nonlinear Funct. Anal. 2013, Article ID 4 (2013)

19. Mahato, NK, Nahak, C: Equilibrium problem under various types of convexities in Banach space. J. Math. Comput. Sci. 1, 77-88 (2011)

20. Qin, X, Cho, SY, Kang, SM: Some results on variational inequalities and generalized equilibrium problems with applications. Comput. Appl. Math. 29, 393-421 (2010)

21. Wu, C, Liu, A: Strong convergence of a hybrid projection iterative algorithm for common solutions of operator equations and of inclusion problems. Fixed Point Theory Appl. 2012, Article ID 90 (2012)

22. Wang, ZM, Zhang, X: Shrinking projection methods for systems of mixed variational inequalities of Browder type, systems of mixed equilibrium problems and fixed point problems. J. Nonlinear Funct. Anal. 2014, Article ID 15 (2014)

23. Qin, X, Shang, M, Su, Y: Strong convergence of a general iterative algorithm for equilibrium problems and variational inequality problems. Math. Comput. Model. 48, 1033-1046 (2008)

24. Jeong, JU: Strong convergence theorems for a generalized mixed equilibrium problem and variational inequality problems. Fixed Point Theory Appl. 2013, 65 (2013)

25. Cho, SY: Iterative processes for common fixed points of two different families of mappings with applications. J. Glob. Optim. 57, 1429-1446 (2013)

26. Rodjanadid, B, Sompong, S: A new iterative method for solving a system of generalized equilibrium problems, generalized mixed equilibrium problems and common fixed point problems in Hilbert spaces. Adv. Fixed Point Theory 3, 675-705 (2013)

27. Wen, DJ, Chen, YA: General iterative methods for generalized equilibrium problems and fixed point problems of k-strict pseudo-contractions. Fixed Point Theory Appl. 2012, 125 (2012)

28. Cho, SY, Qin, X: On the strong convergence of an iterative process for asymptotically strict pseudocontractions and equilibrium problems. Appl. Math. Comput. 235, 430-438 (2014)

29. Hao, Y: On variational inclusion and common fixed point problems in Hilbert spaces with applications. Appl. Math. Comput. 217, 3000-3010 (2010)

30. Liu, LS: Ishikawa and Mann iterative process with errors for nonlinear strongly accretive mappings in Banach spaces. J. Math. Anal. Appl. 194, 114-125 (1995)

31. Suzuki, T: Strong convergence of Krasnoselskii and Mann's type sequences for one-parameter nonexpansive semigroups without Bochner integrals. J. Math. Anal. Appl. 305, 227-239 (2005)

32. Opial, Z: Weak convergence of the sequence of successive approximation for nonexpansive mappings. Bull. Am. Math. Soc. 73, 591-597 (1967)

doi:10.1186/1687-1812-2014-180

Cite this article as: Yuan and Zhang: Convergence of a regularization algorithm for nonexpansive and monotone

operators in Hilbert spaces. Fixed Point Theory and Applications 2014 2014:180. 